\title{
Neurologic Syndromes Predict Higher In-Hospital Mortality in COVID-19
}

Emad Nader Eskandar, MD, MBA, * David J. Altschul, MD,* Rafael de la Garza Ramos, MD, Phillip Cezayirli, MD, Santiago R. Unda, MD, Joshua Benton, BA, Joseph Dardick, BA, Aureliana Toma, MD, Nikunj Patel, BA, Avinash Malaviya, BA, MS, David Flomenbaum, BS, Jenelys Fernandez-Torres, BA, Jenny Lu, BA, Ryan Holland, MD, Elisabetta Burchi, MD, Richard Zampolin, MD, Kevin Hsu, MD, Andrew McClelland, MD, Judah Burns, MD, Amichai Erdfarb, MD, Rishi Malhotra, MD, Michelle Gong, MD, Peter Semczuk, DDS, MPH, Jonathan Gursky, MD, Victor Ferastraoaru, MD, Jillian Rosengard, MD, Daniel Antoniello, MD,

Daniel Labovitz, MD, Charles Esenwa, MD, Mark Milstein, MD, Alexis Boro, MD, and Mark F. Mehler, MD

Neurology ${ }^{\circledR}$ 2021;96:e1527-e1538. doi:10.1212/WNL.0000000000011356

\section{Abstract}

\section{Objective}

Severe acute respiratory syndrome coronavirus 2 (SARS-CoV-2) is protean in its manifestations, affecting nearly every organ system. However, nervous system involvement and its effect on disease outcome are poorly characterized. The objective of this study was to determine whether neurologic syndromes are associated with increased risk of inpatient mortality.

\section{Methods}

A total of 581 hospitalized patients with confirmed SARS-CoV-2 infection, neurologic involvement, and brain imaging were compared to hospitalized non-neurologic patients with coronavirus disease 2019 (COVID-19). Four patterns of neurologic manifestations were identified: acute stroke, new or recrudescent seizures, altered mentation with normal imaging, and neuro-COVID-19 complex. Factors present on admission were analyzed as potential predictors of in-hospital mortality, including sociodemographic variables, preexisting comorbidities, vital signs, laboratory values, and pattern of neurologic manifestations. Significant predictors were incorporated into a disease severity score. Patients with neurologic manifestations were matched with patients of the same age and disease severity to assess the risk of death.

\section{Results}

A total of 4,711 patients with confirmed SARS-CoV-2 infection were admitted to one medical system in New York City during a 6-week period. Of these, 581 (12\%) had neurologic issues of sufficient concern to warrant neuroimaging. These patients were compared to 1,743 nonneurologic patients with COVID-19 matched for age and disease severity admitted during the same period. Patients with altered mentation $(\mathrm{n}=258, p=0.04$, odds ratio [OR] 1.39, confidence interval $[\mathrm{CI}] 1.04-1.86)$ or radiologically confirmed stroke $(\mathrm{n}=55, p=0.001$, OR 3.1, CI 1.65-5.92) had a higher risk of mortality than age- and severity-matched controls.

\section{Conclusions}

The incidence of altered mentation or stroke on admission predicts a modest but significantly higher risk of in-hospital mortality independent of disease severity. While other biomarker factors also predict mortality, measures to identify and treat such patients may be important in reducing overall mortality of COVID-19.

\section{Correspondence}

Dr. Altschul

daltschu@montefiore.org

\section{COVID-19 Resources}

For the latest articles, invited commentaries, and blogs from physicians around the world NPub.org/COVID19

\footnotetext{
*These authors contributed equally to this work.

From the Departments of Neurological Surgery (E.N.E., D.J.A., R.d.I.G.R., P.C., S.R.U., J. Benton, J.D., A.T., J.F.-T., J.L., R.H., E.B.), Neurology (N.P., A. Malaviya, D.F., D.A., D.L., J.G., V.F., J.R., C.E., M.M., A.B., M.F.M.), Radiology (R.Z., K.H., A. McClelland, J. Burns, A.E.), and Critical Care Medicine (R.M., M.G.), Albert Einstein College of Medicine, Montefiore Medical Center (P.S., V.F., J.R.), Bronx, NY.

Go to Neurology.org/N for full disclosures. Funding information and disclosures deemed relevant by the authors, if any, are provided at the end of the article. 


\section{Glossary}

AST $=$ aspartate aminotransferase; $\mathbf{B M I}=$ body mass index; $\mathbf{B U N}=$ blood urea nitrogen; $\mathbf{C I}=$ confidence interval; COVID19 = coronavirus disease 2019; CRP = C-reactive protein; HR = hazard ratio; ICD-10 = International Classification of Diseases-10; INR = international normalized ratio; OR = odds ratio; SARS-CoV-2 = severe acute respiratory syndrome coronavirus 2.

Pulmonary symptoms are the most common in-hospital presentation of severe acute respiratory syndrome coronavirus 2 (SARS-CoV-2) infection. Severely affected patients may have damage to the kidneys, liver, or heart. ${ }^{1,2}$ Since the initial outbreak in Wuhan, China, neurologic involvement has also been described: of 214 cases, $45 \%$ of hospitalized patients presented with headache or dizziness, while $5 \%$ of severely affected patients had a cerebrovascular accident. ${ }^{1}$ Other nervous system manifestations have been identified, including anosmia, increased seizure frequency, recrudescence of stroke symptoms, and Guillain-Barré syndrome. ${ }^{3-8}$ There are reports of large vessel occlusion in younger patients, necrotizing encephalitis, acute demyelinating encephalomyelitis, and meningoencephalitis, although these appear to be relatively rare. $^{9-12}$ Clinical and pathologic studies that have tested for the existence of the SARS-CoV-2 virus in the brain or CSF have yielded variable results. ${ }^{13}$ It remains unclear whether acute neurologic manifestations affect mortality of SARS$\mathrm{CoV}-2$ illness and whether this risk is present in the absence of imaging findings.

The objective of this study was to evaluate factors present on admission - sociodemographic data, medical comorbidities, vital signs, laboratory assessments, and neurologic syndromes-as potential predictors of in-hospital mortality. We hypothesize that clinical evidence of diffuse brain impairment, independent of disease severity and in the absence of imaging findings, is associated with an increased risk of in-hospital mortality. The rationale behind this supposition is that a reduced level of arousal has been previously shown to increase mortality risk from other acute adult medical admission. ${ }^{14}$ Moreover, we suggest that the development of an acute stroke in the context of acute SARS$\mathrm{CoV}-2$ infection also incurs greater risk of mortality independent of age and disease severity. Stroke has been previously shown to be associated with an increased risk of mortality in other infectious disease processes. ${ }^{15}$

\section{Methods}

\section{Standard Protocol Approvals, Registrations, and Patient Consents}

This is a retrospective study of all patients admitted to 4 hospitals within the Montefiore Health System between March 1 and April 16, 2020, with SARS-CoV-2 infection. The study received approval from our institutional ethical standards committee on human experimentation. Written informed consent was waived by our institutional ethical standards committee given the retrospective design of the study. Information on demographics, comorbidities, admission laboratory values, admission medications, admission supplemental oxygen orders, discharge, and mortality was identified through a health care surveillance software package (Clinical Looking Glass; Streamline Health, Atlanta, GA) and review of the primary medical records. ${ }^{16}$

All patients with real-time reverse transcriptase PCRpositive assay testing for SARS-CoV-2 RNA were included. Patients not admitted or who died before admission were excluded because they seldom had a full panel of laboratory studies and full neurologic evaluation could not be assessed. For patients with multiple admissions, only the last reported was considered for analysis. Data were captured on May 7, 2020; therefore, follow-up varied from 3 weeks to 80 days.

The neurologic manifestations cohort consisted of patients exhibiting neurologic conditions of sufficient severity to warrant a neurologically motivated radiographic imaging study-CT, MRI, or diagnostic cerebral angiography-or neurologic consultation. Patients without imaging were placed into the non-neurologic cohort.

We reasoned that different neurologic syndromes may have different prognoses and placed patients with acute stroke confirmed on imaging, new-onset seizures, or recrudescent seizures in patients with epilepsy, and incidental brain lesions not related to SARS-CoV-2 illness, into separate groups. Patients without imaging findings or neurophysiologic abnormalities were divided into those with altered mentation (cognition or arousal) and those with normal mentation but well-documented neurologic signs and symptoms compatible with coronavirus disease 2019 (COVID-19), defined as headache, anosmia, ageusia, chemesthesis, vertigo, presyncope, paresthesias, cranial nerve abnormalities, ataxia, dysautonomia, and skeletal muscle injury.

The stroke cohort was defined as patients with CT angiography or cerebral angiography confirming blockage of an intracranial vessel or CT/MRI findings consistent with acute or subacute infarcts, intracerebral hemorrhage, or subarachnoid hemorrhage. The seizure cohort was based on a careful review of the record by senior epilepsy neurologists, who confirmed the presence of overt seizures or status epilepticus, and the presence or absence of a history of epilepsy. Anatomic brain lesions not related to SARS-CoV-2 illness were defined as the presence of subdural hematoma, brain tumor, chronic infarction, or nonspecific nonvascular territory lesions in the cortex or white matter. All imaging 
findings were established through a review by 2 independent neuroradiologists.

The presence of altered mentation was determined through a review of the notes documenting the history and physical examination generated by emergency department physicians, admitting physicians, or members of the neurology or neurosurgery service when a consultation was requested. Patients were placed into the altered mentation cohort if there was evidence for impaired cognition (defined as disorientation, confusion, agitation, or delirium) or for impaired arousal (defined as drowsiness, somnolence, lethargy, or obtundation). The neuroCOVID-19 complex was defined as normal orientation and arousal with signs and symptoms commonly associated with COVID-19 (including headache, anosmia, ageusia, chemesthesis, vertigo, presyncope, paresthesias, cranial nerve abnormalities, ataxia, dysautonomia, and skeletal muscle injury).

In-hospital deaths and deaths in the National Death Registry were used to collect mortality. Only laboratory values obtained on admission were included. The comorbidities chosen were those used in the Charlson Comorbidity Index using the ICD$10 .{ }^{15,16}$ Every patient's medical record was queried for diagnoses occurring within 5 years of the index admission.

\section{Statistical Analysis}

All analyses were conducted in IBM SPSS (v26.0). For statistical analysis, we represented continuous measurements as means (SD) or as medians (interquartile ranges) and categorical variables as numbers (percentages). ${ }^{17}$ Comparisons between non-neurologic and neurologic groups were performed via 2 -sided $t$ tests, Wilcoxon rank-sum tests, or $\chi^{2}$ testing as appropriate. No data imputation was made for missing values. ${ }^{16}$

Our primary outcome measure was in-hospital mortality. We first performed univariate analysis on potentially predictive candidates. Factors that were significant on initial univariate analysis were then evaluated for independence using multivariate logistic regression.

Our primary analysis utilized a 1:3 matched-control design. For each of the 581 patients with neurologic manifestations, a computer algorithm performed a random search of the remaining 4,130 patients to identify 3 patients having the same age and COVID-19 severity score but no neurologic manifestations, generating a matched-control cohort of 1,743 patients. We then compared the neurologic manifestations cohort to the matched controls. Because each patient had matching controls, subsets of patients within the neurologic manifestations cohort were compared to their respective subsets of controls, thereby maintaining a match in age and severity score. We defined statistical significance as a $p$ value less than 0.05 .

In a second analysis, we used factors found to be independent predictors of in-hospital mortality in the multivariate analysis to estimate hazard ratios (HRs) for death using a Cox proportional hazards model. ${ }^{18}$ The number of days from admission to in-hospital death was used as the time-to-event data. Patients discharged from the hospital were rightcensored. We included 15 independent variables that we considered potentially relevant to in-hospital mortality.

\section{Data Availability}

Data not published within the article are available in a public repository and include digital object identifiers. The anonymized data set is available at Dryad at doi.org/10.5061/dryad. $7 \mathrm{~d} 7 \mathrm{wm} 37 \mathrm{sz}$. Further anonymized data can be shared by request from any qualified investigator.

\section{Results}

During a 6-week period between March 1 and April 16, 2020, a total of 4,711 patients with SARS-CoV-2 infection were hospitalized at 4 hospitals within the Montefiore Health System. Among these, 581 (12\%) individuals had neurologic manifestations and neuroimaging studies. These patients constituted the neurologic manifestations cohort and included those with altered mentation ( $\mathrm{n}=258)$ ( $44 \%$ of the neurologic group and $5.5 \%$ of the total SARS-CoV-2 group), normal mentation with other neurologic signs and symptoms compatible with COVID-19 (neuro-COVID-19 complex) ( $\mathrm{n}=216$ ) (37\% of the neurologic group and $4.6 \%$ of the total SARS-CoV-2 group), stroke ( $n=55$ ) (9\% of the neurologic group and $1.2 \%$ of the total SARS-CoV-2 group), seizures $(n=26)$ ( $4 \%$ of the neurologic group and $0.7 \%$ of the total SARS-CoV-2 group), and other brain lesions $(n=26)$ ( $4 \%$ of the neurologic group and $0.6 \%$ of the total SARS-CoV-2 group).

Out of the 258 patients in the altered mentation group, 61 (23.6\%) had no clear toxic, metabolic disturbances or history of dementia or other premorbid cognitive disturbances. Out of the 55 stroke cases, 36 corresponded to large vessel occlusions, $8(22.2 \%)$ received IV thrombolysis, 12 (33.3\%) underwent endovascular thrombectomy, and 3 exhibited hemorrhagic transformation. Furthermore, 31 (56.4\%) patients of the stroke cohort did not have any underlying comorbidities.

\section{Predictors of Mortality}

We applied univariate analysis across the entire cohort to assess the potential associations with in-hospital mortality of sociodemographics, comorbidities, vital signs, laboratory values, and CNS manifestations (table 1). Among the potential predictors of mortality were male sex, a history of chronic obstructive pulmonary disease, diabetes mellitus, or renal disease, older age, hypoxia, fever, or hypotension, and abnormalities of laboratory values reflecting impairment of the lungs, liver, kidneys, coagulation cascades, and the immune system. ${ }^{16}$

All variables with a $p<0.100$ on the univariate categorical analysis were included in a multivariate logistic regression, which demonstrated that on admission, hypotension, advanced age, elevated serum levels of creatinine or C-reactive 
Table 1 Univariate Analysis of Predictors for Mortality in Patients With Coronavirus Disease 2019 (COVID-19)

\begin{tabular}{|c|c|c|c|}
\hline Variables & Deceased $(n=1,148)$ & Survived $(n=3,563)$ & $p$ Value \\
\hline \multicolumn{4}{|l|}{ Categorical } \\
\hline Male & $674(58.7)$ & $1,837(51.6)$ & $<0.001$ \\
\hline Black & $408(35.5)$ & $1,335(37.5)$ & 0.25 \\
\hline White & $134(11.7)$ & $332(9.3)$ & 0.02 \\
\hline Asian & $38(3.3)$ & $83(2.3)$ & 0.09 \\
\hline Latino & $405(35.3)$ & $1,348(37.8)$ & 0.12 \\
\hline Myocardial infarction & $477(41.5)$ & 249 (6.9) & $<0.001$ \\
\hline Peripheral vascular disease & $135(11.8)$ & $678(19)$ & $<0.001$ \\
\hline Congestive heart failure & $150(13.1)$ & $391(11)$ & 0.08 \\
\hline Cerebrovascular disease & $133(11.6)$ & $373(10.5)$ & 0.35 \\
\hline Dementia & $103(9)$ & $269(7.5)$ & 0.19 \\
\hline Chronic obstructive pulmonary disease & $80(7)$ & $185(5.2)$ & 0.05 \\
\hline Diabetes mellitus complicated & $115(10)$ & $380(10.7)$ & 0.58 \\
\hline Diabetes mellitus simple & $167(14.5)$ & $516(14.5)$ & 0.96 \\
\hline Renal disease & $235(20.5)$ & $598(16.8)$ & 0.01 \\
\hline \multicolumn{4}{|l|}{ Continuous } \\
\hline Age, y & $72.0(13.2)$ & $60.6(16.8)$ & $<0.001$ \\
\hline BMI, $\mathrm{kg} / \mathrm{m}^{2}$ & $26.2(12.0)$ & $28.0(11.4)$ & 0.01 \\
\hline Oxygen saturation, \% & $93(85-97)$ & $95(92-98)$ & $<0.001$ \\
\hline Temperature, ${ }^{\circ} \mathrm{C}$ & $37.1(36.7-37.8)$ & $37.1(36.7-37.1)$ & 0.01 \\
\hline Systolic blood pressure, $\mathrm{mm} \mathrm{Hg}$ & $108(85-133)$ & $125(112-154)$ & $<0.001$ \\
\hline Arterial pressure, $\mathrm{mm} \mathrm{Hg}$ & $76(55-89.7)$ & $89.7(79.7-97)$ & $<0.001$ \\
\hline D-Dimer, mg/L & $2.8(0.27-5.6)$ & $1.4(0.3-2.6)$ & $<0.001$ \\
\hline Platelets, $\mathrm{k} / \mathrm{mm}^{3}$ & $198(149-265.5)$ & $219(161-282)$ & $<0.001$ \\
\hline INR & $1.1(1-1.3)$ & $1.1(1-1.2)$ & $<0.001$ \\
\hline BUN, mg/dL & $33(11-55)$ & $16(9-28)$ & $<0.001$ \\
\hline Creatinine, $\mu \mathrm{mol} / \mathrm{L}$ & $150(100-290)$ & $100(80-150)$ & $<0.001$ \\
\hline Sodium, $\mathrm{mmol} / \mathrm{L}$ & $138(134-142.5)$ & $137(134-140)$ & $<0.001$ \\
\hline Glucose, $\mathrm{mmol} / \mathrm{L}$ & $7.4(6.2-10.3)$ & $8.6(6.7-12.8)$ & 0.31 \\
\hline AST, U/L & $50(29-78)$ & $37(23-58)$ & $<0.001$ \\
\hline ALT, U/L & $28(16-44)$ & $26(15-43)$ & 0.73 \\
\hline WBC count per $\mathrm{mm}^{3}$ & $8,100(5,800-11,300)$ & $7,200(5,300-9,900)$ & 0.001 \\
\hline Lymphocytes per $\mathrm{mm}^{3}$ & $900(600-1,300)$ & $1,000(700-1,500)$ & $<0.001$ \\
\hline Interleukin-6, pg/mL & $81.4(36.8-183)$ & $29.7(12.8-63.2)$ & $<0.001$ \\
\hline Ferritin, $\mu \mathrm{g} / \mathrm{L}$ & $1,032(205-1,597)$ & $648(156-1,060)$ & $<0.001$ \\
\hline CRP, mg/L & $15.4(2.9-22.6)$ & $7.1(0.6-13.9)$ & $<0.001$ \\
\hline Procalcitonin, ng/mL & $0.7(0.2-3.2)$ & $0.1(0.1-0.5)$ & $<0.001$ \\
\hline Troponin, ng/mL & $0.02(0.01-0.07)$ & $0.01(0.01-0.01)$ & $<0.001$ \\
\hline
\end{tabular}


Table 1 Univariate Analysis of Predictors for Mortality in Patients With Coronavirus Disease 2019 (COVID-19) (continued)

\begin{tabular}{|c|c|c|c|}
\hline Variables & Deceased $(n=1,148)$ & Survived $(n=3,563)$ & $p$ Value \\
\hline \multicolumn{4}{|l|}{ Neurologic manifestations } \\
\hline All & $199(17.3)$ & $382(10.7)$ & $<0.001$ \\
\hline Altered mentation & $104(9.1)$ & $154(4.3)$ & $<0.001$ \\
\hline Stroke & $27(2.4)$ & $28(0.8)$ & $<0.001$ \\
\hline Seizure & $5(0.4)$ & $21(0.7)$ & 1.00 \\
\hline Neuro-COVID-19 complex & $58(5.1)$ & $158(4.4)$ & 0.37 \\
\hline Other brain anatomic lesions & $5(0.4)$ & $21(0.6)$ & 0.65 \\
\hline \multicolumn{4}{|c|}{ 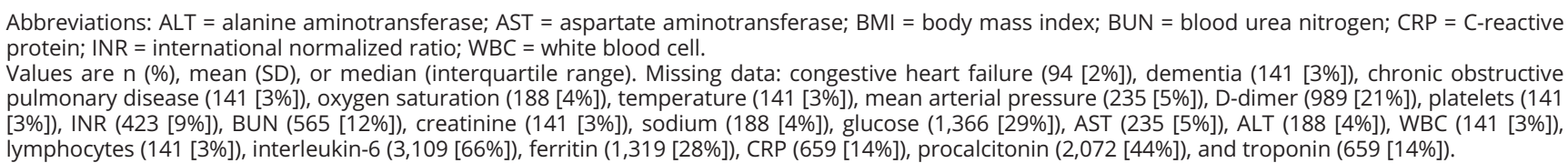 } \\
\hline
\end{tabular}

protein (CRP), hypoxia, reduced troponin, platelet count, increased aspartate aminotransferase (AST), deranged sodium, reduced lymphocyte count, high body mass index (BMI), and male sex were significant independent predictors for an increased risk of in-hospital mortality (table 2). ${ }^{19}$ Findings consistent with acute stroke $(p<0.001$, odds ratio [OR] 3.49, confidence interval [CI] 2.9-4.1) and altered mentation $(p=0.002$, OR 1.61, CI 1.3-1.9) were significant predictors for an increased risk of in-hospital mortality independent of the other factors (table 2).

\section{Predictors of Neurologic Manifestations}

We also compared the incidence of the various measures as potential correlates of neurologic manifestations. There was a significant association between altered mentation and increasing age, Black race, Latino ethnicity, history of stroke, chronic obstructive pulmonary disease, congestive heart failure, and renal disease. Other significant correlates were mean arterial pressure $<70 \mathrm{~mm} \mathrm{Hg}$, D-dimer $>3 \mathrm{mg} / \mathrm{L}$, platelets $<150,000$ per $\mathrm{mm}^{3}{ }^{3}$ international normalized ratio (INR) $>1.2$, blood urea nitrogen $(\mathrm{BUN})>30 \mathrm{mg} / \mathrm{dL}$, creatinine $>1.5 \mu \mathrm{mol} / \mathrm{L}$, procalcitonin $>0.1 \mathrm{ng} / \mathrm{ml}$, and troponin $>0.1$ $\mathrm{ng} / \mathrm{mL}$ (table 3 ). Patients with stroke had similar characteristics to the larger non-neurologic cohort except for a significantly higher percentage of patients with $\mathrm{D}$-dimer levels $>3$ $\mathrm{mg} / \mathrm{L}$ ( $55 \%$ vs $30 \%, p=0.001,95 \%$ CI 1.6-5.0, table 4 ). Being younger $(<60$ years [OR $0.454,95 \%$ CI $0.23-0.895, p=$ $0.03]$ ) and having IL-6 levels over $150 \mathrm{pg} / \mathrm{ml}$ (OR 4.959, 95\% CI 1.324-18.576, $p=0.027$ ) were independent predictors of seizures in patients with COVID-19 (table e-1) [available from Dryad: doi.org/10.5061/dryad.7d7wm37sz]). ${ }^{16}$

\section{Disease Severity Score}

In order to test the hypothesis that altered mentation or stroke carry an additional risk of in-hospital mortality, we sought to account for underlying disease severity in the most straightforward fashion possible. On univariate analysis, 24 factors had a significant association with mortality (table 1), while multivariate regression demonstrated that 14 of these were independent predictors of in-hospital mortality on admission: increasing age, hypotension, hypoxemia, elevated serum levels of creatinine, CRP, and D-dimer, and relative thrombocytopenia (table 2).

Based on the respective ORs of these variables, we created a scoring system reflecting underlying disease severity. ${ }^{16}$ Our goal was to capture the impairment in multiple organ systems without overfitting the data. The score included (1) age by decile, so that patients above 60,70 , or 80 received 1,2 , or 3 points, respectively; (2) hypotension, so that calculated mean arterial pressure (MAP) below 80,70 , and 60 received 1,2 , or 3 points, respectively; (3) impaired pulmonary function, reflected in oxygen saturations below $94 \%$, received 1 point; (4) impaired renal function, reflected in BUN greater than 30, received 1 point; (5) coagulopathy, reflected as an INR greater than 1.2 and increased inflammatory response, reflected in CRP levels greater than 10, received 1 point. The maximum score was 10 points (table e-2 [available from Dryad: doi.org/10.5061/dryad.7d7wm37sz]).

This scoring system was applied to the entire cohort, and the majority of patients were distributed in the first 3 points of the score and, moreover, the severity score corresponded to a linear increase for in-hospital mortality over the range of $0-10$ points (figure).

\section{Matched Case-Control Analysis}

For each of the 581 patients with neurologic manifestations, a random search algorithm identified 3 age- and severity-matched patients, generating a cohort of 1,180 controls. Because age and severity score were deliberately matched, the distribution of age and disease severity was the same in the CNS groups and the matched-control group. Other variables were not explicitly matched, and exhibited small differences (table 5). 
Table 2 Multivariate Logistic Regression Analysis of In-Hospital Mortality Predictors in Patients With Coronavirus Disease 2019 (COVID-19)

\begin{tabular}{|c|c|c|c|}
\hline Predictors & $p$ Value & OR & $95 \% \mathrm{Cl}$ \\
\hline Mean arterial pressure $<70 \mathrm{~mm} \mathrm{Hg}$ & $<0.001$ & 17.57 & $17.2-17.9$ \\
\hline Stroke & $<0.001$ & 3.49 & $2.9-4.1$ \\
\hline Age $>65$ years & $<0.001$ & 3.31 & $3.1-3.5$ \\
\hline Creatinine $>150 \mu \mathrm{mol} / \mathrm{L}$ & $<0.001$ & 1.77 & $1.6-2.0$ \\
\hline C-reactive protein $>10 \mathrm{mg} / \mathrm{L}$ & $<0.001$ & 1.64 & $1.5-1.8$ \\
\hline Altered mentation & 0.002 & 1.61 & $1.3-1.9$ \\
\hline Oxygen saturation $<94 \%$ & $<0.001$ & 1.59 & $1.4-1.8$ \\
\hline Troponin $<0.1 \mathrm{ng} / \mathrm{mL}$ & 0.005 & 1.43 & $1.2-1.7$ \\
\hline Platelets $<150,000$ per $\mathrm{mm}^{3}$ & $<0.001$ & 1.43 & $1.2-1.6$ \\
\hline Aspartate aminotransferase $>40 \mathrm{U} / \mathrm{L}$ & $<0.001$ & 1.41 & $1.2-1.6$ \\
\hline Hyponatremia or hypernatremia & 0.023 & 1.30 & $1.1-1.5$ \\
\hline Lymphocyte count $<1,000$ per $\mathrm{mm}^{3}$ & 0.01 & 1.23 & $1.1-1.4$ \\
\hline $\mathrm{BMI}>30 \mathrm{~kg} / \mathrm{m}^{2}$ & 0.03 & 1.22 & $1.0-1.4$ \\
\hline Male sex & 0.04 & 1.18 & $1.0-1.3$ \\
\hline Renal disease & 0.06 & 1.21 & $1.0-1.4$ \\
\hline Temperature $>38^{\circ} \mathrm{C}$ & 0.06 & 1.21 & $1.0-1.4$ \\
\hline International normalized ratio $>1.2$ & 0.10 & 1.18 & $1.0-1.4$ \\
\hline D-dimer $>3 \mathrm{mg} / \mathrm{L}$ & 0.15 & 1.14 & $1.0-1.3$ \\
\hline Blood urea nitrogen $>30 \mathrm{mg} / \mathrm{dL}$ & 0.43 & 1.09 & $0.9-1.3$ \\
\hline Neuro-COVID-19 complex & 0.85 & 1.04 & $0.7-1.4$ \\
\hline White blood cell count $>10.8$ or $<4.1 \mathrm{k} / \mathrm{mm}^{3}$ & 0.53 & 0.86 & $0.4-1.4$ \\
\hline
\end{tabular}

Abbreviations: $\mathrm{BMI}=$ body mass index; $\mathrm{Cl}$ = confidence interval; $\mathrm{OR}=$ odds ratio.

Within the neurologic manifestation groups, patients with stroke had the highest risk of in-hospital mortality, which was significantly higher than matched controls ( $49 \%$ vs $24 \%, p=$ 0.001, OR 3.1, 95\% CI 1.65-5.92). The same was true for patients with altered mentation but the absence of imaging abnormalities ( $40 \%$ vs $33 \%$, respectively, $p=0.04$, OR 1.39 , 95\% CI 1.04-1.86). There was a trend for patients presenting with impaired arousal to have a higher risk of mortality compared with those with impaired cognition, although this was not statistically significant. There was no significant increase in risk for patients with new or recurrent seizures, neuro-COVID-19 complex, or those with incidentally discovered brain lesions (table 5).

\section{Cox Proportional Hazards Model}

From the original set of 40 potential predictors of mortality, univariate analysis identified 24 that exhibited a significant correlation, and multivariate regression identified 14 as independent predictors of mortality. They also appear to combine in a broadly additive manner as demonstrated in the severity score and matched-control analysis. These considerations informed the choice of predictor variables in a Cox proportional hazards model. We included advanced age, male sex, and elevated BMI as all have been previously associated with poor clinical outcomes. We included blood pressure on admission, as it had the largest OR on multivariate analysis. We included platelets, given the potential role of coagulopathy in stroke, creatinine to capture kidney damage, AST to capture liver damage, CRP to capture inflammatory derangement, troponin to capture cardiac injury, along with lymphocyte count to capture immune system dysfunction. We also included stroke, altered mentation, and neuroCOVID-19 complex as potential predictors. Time to event was the time from admission to in-hospital death. Patients discharged from the hospital were right-censored.

Multiple factors were found independently associated with inhospital mortality: hypotension $(p<0.0001$, HR 4.39, CI $4.2-4.5)$, older age ( $p<0.001, \mathrm{HR} 2.61, \mathrm{CI} 2.5-2.7)$, hypoxia ( $p<$ 0.001, HR 1.43, CI 1.3-1.5), elevated creatinine $(p<0.001$, HR 1.5, CI 1.4-1.6), elevated CRP ( $p<0.001$, HR 1.42 CI 1.3-1.6), 
Table 3 Distribution of Variables in Severe Acute Respiratory Syndrome Coronavirus 2 (SARS-CoV-2)-Positive Patients With Altered Mentation

\begin{tabular}{|c|c|c|c|c|}
\hline Variable & Altered mentation ( $n=258), n(\%)$ & Non-neuro $(n=4,130), n(\%)$ & $p$ Value & OR $(95 \% \mathrm{Cl})$ \\
\hline Male & $144(56)$ & $2,367(53)$ & 0.44 & $1.11(0.9-1.4)$ \\
\hline Black & $119(46)$ & $1,624(36)$ & 0.002 & $1.49(1.2-1.9)$ \\
\hline White & $29(11)$ & $437(10)$ & 0.45 & $1.16(0.8-1.7)$ \\
\hline Asian & $8(3)$ & $113(3)$ & 0.54 & $1.23(0.6-2.5)$ \\
\hline Latino & $75(29)$ & $1,678(38)$ & 0.01 & $0.68(0.5-0.9)$ \\
\hline Myocardial infarction & $40(16)$ & $686(15)$ & 0.93 & $1.01(0.7-1.4)$ \\
\hline PVD & $30(12)$ & $783(18)$ & 0.01 & $0.61(0.4-0.9)$ \\
\hline Congestive heart failure & $42(16)$ & $499(11)$ & 0.02 & $1.51(1.1-2.1)$ \\
\hline Cerebrovascular disease & $38(15)$ & $468(11)$ & 0.05 & $1.45(1-2.1)$ \\
\hline Dementia & $23(9)$ & $349(8)$ & 0.64 & $1.12(0.7-1.7)$ \\
\hline COPD & $25(10)$ & $240(6)$ & 0.01 & $1.83(1.2-2.8)$ \\
\hline DM complicated & $29(11)$ & $466(10)$ & 0.68 & $1.08(0.7-1.6)$ \\
\hline All diabetes & $44(17)$ & $639(14)$ & 0.24 & $1.23(0.9-1.7)$ \\
\hline Renal disease & $62(24)$ & $771(17)$ & 0.01 & $1.51(1.1-2)$ \\
\hline Age $>60$ years & $220(74)$ & $2,765(47)$ & $<0.001$ & $3.53(2.5-5)$ \\
\hline $\mathrm{BMI}>30 \mathrm{~kg} / \mathrm{m}^{2}$ & $227(32)$ & $4,065(41)$ & 0.07 & $0.70(0.5-1)$ \\
\hline Oxygen saturation $<94 \%$ & $87(36)$ & $1,631(38)$ & 0.46 & $0.90(0.7-1.2)$ \\
\hline Temperature $>38^{\circ} \mathrm{C}$ & $31(13)$ & $823(19)$ & 0.01 & $0.62(0.4-0.9)$ \\
\hline MAP $<70 \mathrm{~mm} \mathrm{Hg}$ & $51(21)$ & $532(13)$ & $<0.001$ & $1.86(1.4-2.6)$ \\
\hline D-dimer > $3 \mu \mathrm{g} / \mathrm{mL}$ & $81(41)$ & $1,070(30)$ & 0.003 & $1.58(1.2-2.1)$ \\
\hline Platelets $<150 \times 10^{3} / \mu \mathrm{L}$ & $55(22)$ & $836(19)$ & 0.28 & $1.19(0.9-1.6)$ \\
\hline INR $>1.2$ & $70(30)$ & $747(18)$ & $<0.001$ & $1.89(1.4-2.5)$ \\
\hline BUN $>30 \mathrm{mg} / \mathrm{dL}$ & $113(53)$ & $1,172(30)$ & $<0.001$ & $2.67(2-3.5)$ \\
\hline Creatinine $>1.5 \mathrm{mg} / \mathrm{dL}$ & $131(53)$ & $1,318(30)$ & $<0.001$ & $2.54(2-3.3)$ \\
\hline Sodium $<139$ or $>154 \mathrm{mEq} / \mathrm{L}$ & $34(14)$ & $558(13)$ & 0.69 & $1.08(0.7-1.6)$ \\
\hline Glucose $<60$ or $>500 \mathrm{mEq} / \mathrm{L}$ & $10(6)$ & $104(3)$ & 0.13 & $1.76(0.9-3.4)$ \\
\hline AST $>40 \mathrm{U} / \mathrm{L}$ & $120(50)$ & $2,001(47)$ & 0.47 & $1.11(0.9-3.4)$ \\
\hline ALT $>40 \mathrm{U} / \mathrm{L}$ & $55(22)$ & $1,237(29)$ & 0.03 & $0.70(0.5-1)$ \\
\hline WBC $<4,800$ or $>10,800 / \mu \mathrm{L}$ & $214(86)$ & $3,677(85)$ & 0.65 & $1.10(0.8-1.6)$ \\
\hline Lymphocytes $<1,000 / \mu \mathrm{L}$ & $125(50)$ & $1,999(46)$ & 0.22 & $1.18(0.9-1.5)$ \\
\hline Interleukin-6 >150 pg/mL & $20(17)$ & $271(14)$ & 0.42 & $1.23(0.7-2)$ \\
\hline Ferritin $>300 \mathrm{ng} / \mathrm{mL}$ & $140(79)$ & $2,421(75)$ & 0.33 & $1.21(0.8-1.7)$ \\
\hline C-reactive protein $>1 \mathrm{mg} / \mathrm{dL}$ & $90(43)$ & $1,763(47)$ & 0.26 & $0.84(0.6-1.1)$ \\
\hline Procalcitonin $>0.1 \mathrm{ng} / \mathrm{mL}$ & $109(71)$ & $1,615(55)$ & $<0.001$ & $2.02(1.4-2.9)$ \\
\hline Troponin $>0.1 \mathrm{ng} / \mathrm{mL}$ & $46(20)$ & $404(11)$ & $<0.001$ & $2.08(1.5-2.9)$ \\
\hline
\end{tabular}

Abbreviations: $\mathrm{ALT}=$ alanine aminotransferase; $\mathrm{AST}=$ aspartate aminotransferase; $\mathrm{BMI}=$ body mass index; $\mathrm{BUN}=$ blood urea nitrogen; $\mathrm{Cl}=$ confidence interval; COPD = chronic obstructive pulmonary disease; $\mathrm{DM}=$ diabetes mellitus; INR = international normalized ratio; $\mathrm{MAP}=$ mean arterial pressure; OR = odds ratio; PVD = peripheral vascular disease; $\mathrm{WBC}=$ white blood cell. 
Table 4 Distribution of Variables in Severe Acute Respiratory Syndrome Coronavirus 2 (SARS-CoV-2)-Positive Patients With Stroke

\begin{tabular}{|c|c|c|c|c|}
\hline Variable & Stroke $(n=58), n(\%)$ & Non-neuro $(n=4,130), n(\%)$ & $p$ Value & OR $(95 \% \mathrm{Cl})$ \\
\hline Male & $27(49)$ & $2,367(53)$ & 0.587 & $0.84(0.5-1.4)$ \\
\hline Black & $17(31)$ & $1,624(36)$ & 0.400 & $0.76(0.4-1.3)$ \\
\hline White & $4(7)$ & $437(10)$ & 0.653 & $0.71(0.3-2)$ \\
\hline Asian & $1(2)$ & $113(3)$ & 1.000 & $0.70(0.1-5.1)$ \\
\hline Latino & $20(36)$ & $1,678(38)$ & 1.000 & $0.96(0.6-1.7)$ \\
\hline Myocardial infarction & $17(31)$ & $686(15)$ & 0.004 & $2.49(1.4-4.4)$ \\
\hline PVD & $3(5)$ & $783(18)$ & 0.018 & $0.27(0.1-0.9)$ \\
\hline Congestive heart failure & $5(9)$ & $499(11)$ & 0.676 & $0.75(0.3-1.9)$ \\
\hline Cerebrovascular disease & $9(16)$ & $468(10)$ & 0.190 & $1.61(0.8-3.3)$ \\
\hline Dementia & $4(7)$ & $349(8)$ & 1.000 & $0.88(0.3-2.5)$ \\
\hline COPD & $5(9)$ & $240(5)$ & 0.249 & $1.64(0.6-4.1)$ \\
\hline DM complicated & $8(15)$ & $466(10)$ & 0.371 & $1.46(0.7-3.1)$ \\
\hline All diabetes & $8(15)$ & $639(14)$ & 1.000 & $1.0(0.5-2.1)$ \\
\hline Renal disease & $12(22)$ & $771(17)$ & 0.476 & $1.30(0.7-2.5)$ \\
\hline Age $>60$ years & $50(91)$ & $4,065(91)$ & 0.815 & $0.98(0.4-2.5)$ \\
\hline $\mathrm{BMI}>30 \mathrm{~kg} / \mathrm{m}^{2}$ & $37(67)$ & $2,765(61)$ & 0.577 & $1.19(0.7-2.1)$ \\
\hline Oxygen saturation $<94 \%$ & $18(34)$ & $1,631(39)$ & 0.670 & $0.84(0.5-1.5)$ \\
\hline Temperature $>38^{\circ} \mathrm{C}$ & $8(15)$ & $823(20)$ & 0.598 & $0.75(0.4-1.6)$ \\
\hline MAP $<70 \mathrm{~mm} \mathrm{Hg}$ & $10(19)$ & $532(12)$ & 0.214 & $1.57(0.8-3.1)$ \\
\hline D-dimer $>3 \mu \mathrm{g} / \mathrm{mL}$ & $26(55)$ & $1,070(30)$ & 0.001 & $2.81(1.6-5)$ \\
\hline Platelets $<150 \times 10^{3} / \mu \mathrm{L}$ & $9(17)$ & $836(19)$ & 0.730 & $0.83(0.4-1.7)$ \\
\hline INR $>1.2$ & $12(24)$ & $747(18)$ & 0.472 & $1.31(0.7-2.5)$ \\
\hline BUN $>30 \mathrm{mg} / \mathrm{dL}$ & $14(30)$ & $1,172(29)$ & 1.000 & $0.98(0.5-1.8)$ \\
\hline Creatinine $>1.5 \mathrm{mg} / \mathrm{dL}$ & $15(28)$ & $1,318(30)$ & 0.659 & $0.83(0.5-1.5)$ \\
\hline Sodium $<139$ or $>154 \mathrm{mEq} / \mathrm{L}$ & $6(12)$ & $558(13)$ & 1.000 & $0.87(0.4-2)$ \\
\hline Glucose $<60$ or $>500 \mathrm{mEq} / \mathrm{L}$ & $2(5)$ & $104(3)$ & 0.375 & $1.58(0.4-6.6)$ \\
\hline AST $>40 \mathrm{U} / \mathrm{L}$ & $26(53)$ & $2,001(47)$ & 0.473 & $1.26(0.7-2.2)$ \\
\hline ALT $>40 \mathrm{U} / \mathrm{L}$ & $12(24)$ & $1,237(29)$ & 0.533 & $0.77(0.4-1.5)$ \\
\hline WBC $<4,800$ or $>10,800 / \mu \mathrm{L}$ & $47(87)$ & $3,677(85)$ & 0.848 & $1.21(0.5-2.7)$ \\
\hline Lymphocytes $<1,000 / \mu \mathrm{L}$ & $24(44)$ & $1,999(46)$ & 0.891 & $0.93(0.5-1.6)$ \\
\hline Interleukin- $6>150 \mathrm{pg} / \mathrm{mL}$ & $2(13)$ & $271(14)$ & 1.000 & $0.87(0.2-3.9)$ \\
\hline Ferritin $>300 \mathrm{ng} / \mathrm{mL}$ & $33(80)$ & $2,421(75)$ & 0.584 & $1.35(0.6-2.9)$ \\
\hline C-reactive protein $>1 \mathrm{mg} / \mathrm{dL}$ & $25(54)$ & $1,763(47)$ & 0.373 & $1.34(0.8-2.4)$ \\
\hline Procalcitonin $>0.1 \mathrm{ng} / \mathrm{mL}$ & $19(58)$ & $1,615(55)$ & 0.862 & $1.07(0.5-2.2)$ \\
\hline Troponin $>0.1 \mathrm{ng} / \mathrm{mL}$ & $7(14)$ & $404(10)$ & 0.488 & $1.35(0.6-3)$ \\
\hline
\end{tabular}

Abbreviations: $\mathrm{ALT}=$ alanine aminotransferase; $\mathrm{AST}=$ aspartate aminotransferase; $\mathrm{BMI}=$ body mass index; $\mathrm{BUN}=$ blood urea nitrogen; $\mathrm{Cl}=$ confidence interval; COPD = chronic obstructive pulmonary disease; DM = diabetes mellitus; INR = international normalized ratio; MAP = mean arterial pressure; OR = odds ratio; PVD = peripheral vascular disease; WBC = white blood cell. 

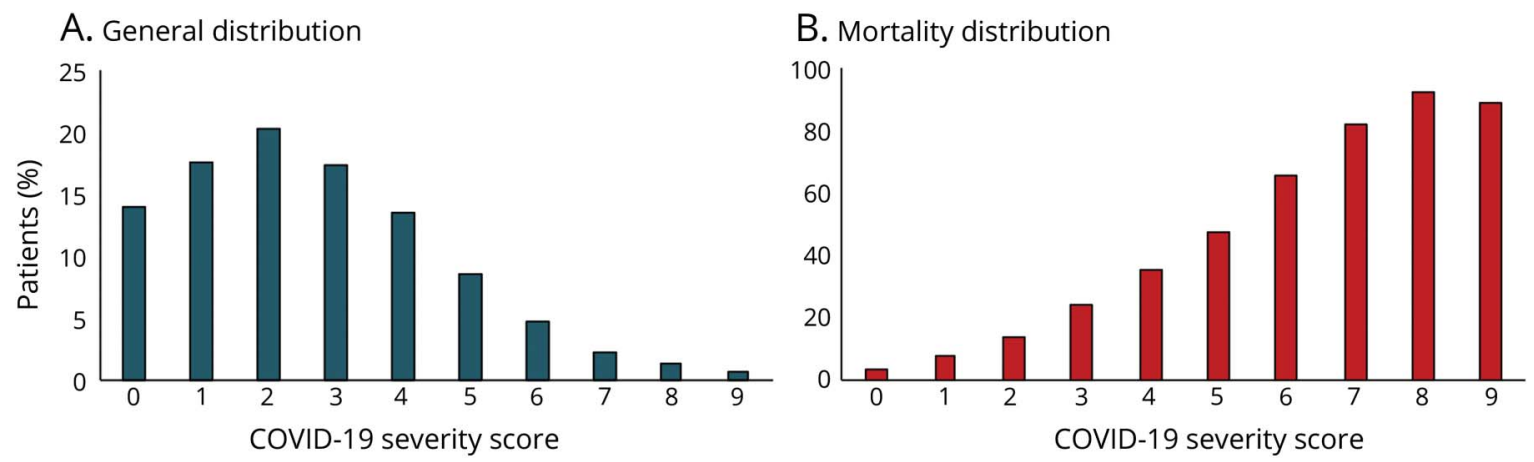

(A) General distribution. (B) Mortality distribution.

lymphocytopenia ( $p<0.00$, HR 1.37 , CI $1.2-1.5$ ), elevated troponin ( $p=0.001$, HR 1.34, CI 1.2-1.5), hyponatremia or hypernatremia ( $p=0.01$, HR 1.23 , CI 1.1-1.4), thrombocytopenia $(p=0.003$, HR 1.23, CI 1.1-1.4), and elevated AST ( $p=$ 0.01 , HR 1.17, CI 1.0-1.3) (table 6). Altered mentation was a significant independent predictor of in-hospital mortality $(p<$ 0.003, HR 1.37, CI 1.2-1.6), with the HR suggesting that the risk is similar to that imposed by cardiac injury. Stroke is also a significant predictor of mortality ( $p=0.004$, HR 1.75 , CI 1.4-2.1), with the HR between hypoxia and age older than 65 .

\section{Discussion}

We present the largest inpatient cohort of SARS-CoV-2infected patients to date, evaluating predictors of inpatient mortality as they relate to neurologic syndromes at presentation. The neurologic findings seen in this cohort were similar to other large cohort studies. ${ }^{6,20,21}$ A small but substantial subset of patients had associated neurologic presentations of sufficient severity to warrant imaging of the neuraxis. Although the majority had normal neuroimaging, the distinction between those with altered cognition or arousal and those exhibiting other neurologic signs and symptoms associated with COVID-19 infection (neuro-COVID-19 complex) appears to be important.

The etiology of altered mentation in COVID-19 is not clear. In this study, there was an association between altered mentation and hypotension, renal impairment as demonstrated by elevated BUN and creatinine, disturbed coagulation as evidenced by elevated D-dimer levels, prolonged INR and reduced platelet counts, and increased inflammation as evidenced by elevated levels of procalcitonin. These biomarker abnormalities are associated with multiorgan system failure and more severe SARS-CoV-2 illness. These patients were less likely to have traditional symptoms such as fever or decreased oxygen saturation. It is unclear whether these findings are exclusive to COVID-19 infection. Other studies have shown that delirium is a predictor for increased inpatient mortality irrespective of underlying diagnosis, particularly in elderly patients. ${ }^{22}$ Even when controlling for biomarker abnormalities, patients with impaired cognition or arousal without abnormal neuroimaging findings exhibited an increased risk of inpatient mortality, suggesting that other yet-to-be determined mechanisms may be

Table 5 In-Hospital Mortality in Patients With Neurologic Manifestations and Matched Controls

\begin{tabular}{|c|c|c|c|c|c|c|}
\hline \multirow[b]{2}{*}{ Neurologic manifestations } & \multicolumn{2}{|c|}{ Neurologic cohort, n (\%) } & \multicolumn{2}{|c|}{ Matched controls, $\mathrm{n}(\%)$} & \multirow[b]{2}{*}{$p$ Value } & \multirow[b]{2}{*}{ OR $(95 \% \mathrm{Cl})$} \\
\hline & Admitted & Deceased & Admitted & Deceased & & \\
\hline All neurologic & 581 & $199(34)$ & 1,743 & $506(29)$ & 0.02 & $1.27(1.04-1.56)$ \\
\hline Altered mentation & 258 & $104(40)$ & 774 & $253(33)$ & 0.04 & $1.39(1.04-1.86)$ \\
\hline Stroke & 55 & $27(49)$ & 165 & $39(24)$ & 0.001 & $3.1(1.65-5.92)$ \\
\hline Neuro-COVID-19 complex & 216 & $58(27)$ & 648 & $173(27)$ & 0.85 & $1.0(0.7-1.42)$ \\
\hline Seizure & 26 & $5(19)$ & 78 & $13(17)$ & 0.77 & $1.26(0.4-3.98)$ \\
\hline Incidental brain lesion & 26 & $5(19)$ & 78 & $24(30)$ & 0.36 & $0.54(0.2-1.6)$ \\
\hline
\end{tabular}

Abbreviations: $\mathrm{Cl}=$ confidence interval; COVID-19 = coronavirus disease 2019; OR = odds ratio.

For each neurologic patient, there are 3 control patients matched for age and COVID-19 disease severity score. 
Table 6 Cox Regression Analysis for Mortality Outcomes

\begin{tabular}{|c|c|c|c|}
\hline Predictors & $p$ Value & HR & $\mathrm{Cl}$ \\
\hline MAP $<70 \mathrm{~mm} \mathrm{Hg}$ & $<0.001$ & 4.39 & $4.2-4.5$ \\
\hline Age $>65$ years & $<0.001$ & 2.61 & $2.5-2.7$ \\
\hline Stroke & 0.004 & 1.75 & $1.4-2.1$ \\
\hline Creatinine $>150 \mu \mathrm{mol} / \mathrm{L}$ & $<0.001$ & 1.50 & $1.4-1.6$ \\
\hline Oxygen saturation <94\% & $<0.001$ & 1.43 & $1.3-1.5$ \\
\hline C-reactive protein >10 mg/L & $<0.001$ & 1.42 & $1.3-1.6$ \\
\hline Lymphocyte count $<1,000$ per $\mathrm{mm}^{3}$ & $<0.001$ & 1.37 & $1.2-1.5$ \\
\hline Altered mentation & 0.003 & 1.37 & $1.2-1.6$ \\
\hline Troponin >0.1 ng/mL & 0.001 & 1.34 & $1.2-1.5$ \\
\hline Hyponatremia or hypernatremia & 0.01 & 1.23 & $1.1-1.4$ \\
\hline Platelets $<150,000$ per $\mathrm{mm}^{3}$ & 0.003 & 1.23 & $1.1-1.4$ \\
\hline AST $>40 \mathrm{U} / \mathrm{L}$ & 0.01 & 1.17 & $1.0-1.3$ \\
\hline $\mathrm{BMI}>30 \mathrm{~kg} / \mathrm{m}^{2}$ & 0.19 & 1.09 & $1.0-1.2$ \\
\hline Male sex & 0.24 & 1.07 & $1.0-1.2$ \\
\hline
\end{tabular}

Abbreviations: $\mathrm{AST}=$ aspartate aminotransferase; $\mathrm{BMI}=$ body mass index; $\mathrm{Cl}$ = confidence interval; HR = hazard ratio; MAP = mean arterial pressure.

at play. Irrespective of the etiologic factors, such neurologic presentations can be subtle but important indications of more severe SARS-CoV-2 illness and should be taken seriously in hospital emergency departments. While other biomarker findings such as hypotension, D-dimer, coagulopathy, and renal failure may be more predictive of illness severity, and mortality, neurologic syndromes portend a higher risk of mortality, and can be easily assessed early in a patient encounter. The biomarkers we found to be most correlative with poor outcome and potentially modifiable such as BUN, INR, oxygen saturation, mean arterial pressure, and CRP could serve as potential targets for future research for treatments or management paradigms. $^{16}$

Stroke with concomitant SARS-CoV-2 infection is a rare but serious complication of the illness. ${ }^{12}$ In our cohort, stroke represented $9 \%$ of our neurologic cohort and $1 \%$ of all SARSCoV-2-infected patients. This subgroup was more likely to have elevated D-dimer and CRP compared with controls. Stroke with SARS-CoV-2 infection had an even higher risk of inpatient mortality and these individuals likely represent a more severe manifestation of the illness. The etiology of stroke and SARS-CoV-2 infection is likely multifactorial, with some that can be attributed to the unique COVID-19 coagulopathy, severe systemic inflammatory reactions, and patients with risk factors for stroke in which the illness can be a trigger. We discovered that more than half of the patients with stroke did not have underlying risk factors, which is highly unusual. This suggests like in other studies that SARS-CoV-2 infection is itself a risk factor for stroke. ${ }^{23}$ During the time period of the study, all patients with acute stroke were managed according to our institutional protocols and policies. Despite other changes to hospital throughput, the neurologic services remained stable. An important caveat to note is that all patients that carry the diagnosis of acute ischemic stroke carry an increased risk of mortality compared to those without stroke on admission. ${ }^{24}$ The mortality risk therefore may be intrinsically predicated with the stroke diagnosis as compared to the SARS-CoV-2 infection itself.

Due to the retrospective nature of this study, there are potential limitations to this study. The majority of patients were a minority urban population and occurring at an epoch during a major surge period of the pandemic. This may bias the results toward higher mortality, as this was a great strain on treating hospitals at the time. This study was limited to evaluating inpatient mortality, therefore any deaths that occurred outside of our health care system may have been lost. The health system during this epoch was over total capacity for critical care beds but not over capacity for beds, which may have limited the number of patients receiving neurologic consultation, adequate neurologic examination documentation, and neurologic imaging. It is also possible that minor stroke cases could have been missed in severe SARS-CoV-2 illness. Therefore, this may underestimate the true number of patients with neurologic manifestations found in our cohort. Despite this, however, using a matched analysis, neurologic symptoms alone still predicted higher inpatient mortality. We therefore hypothesize that in a prospective study, during nonpandemic volumes, the predictive effect of neurologic manifestations on mortality may be greater than perceived in this study.

Unlike other studies, in our institution we did not find significant differences in mortality in our Black and Latinx populations when controlling for underlying comorbid illness. $^{25-27}$ This further suggests that variations in outcome based on race and ethnicity are less tied to bias within health care and more likely a result of structural and systemic racism, leading to inequity of health as it pertains to comorbid conditions, and overall health of populations. ${ }^{28}$ These findings correspond in the neurologic realm as with SARS-CoV-2 infection itself.

To our knowledge, this is the largest study analyzing the neurologic manifestations of COVID-19 and their effect on mortality. Documented CNS manifestations such as encephalopathy, stroke, seizure, and syncope are relatively common, being present in at least $13 \%$ of hospitalized patients with COVID-19, although the incidence is likely much higher. Within the spectrum of CNS manifestations, altered mentation and stroke confer a higher risk of mortality above the severity of underlying illness. The presence of these syndromes may represent a different clinically important syndromic expression of SARS-Cov-2 infection that carries a greater risk of mortality and may benefit from targeted treatment. 


\section{Study Funding}

There were no sources of funding for this work.

\section{Disclosure}

None of the authors has any relevant financial conflicts to disclose. Go to Neurology.org/N for full disclosures.

\section{Publication History}

Received by Neurology August 18, 2020. Accepted in final form November 24, 2020.

Appendix Authors

\begin{tabular}{|c|c|c|}
\hline Name & Location & Contribution \\
\hline $\begin{array}{l}\text { Emad Nader } \\
\text { Eskandar, MD, } \\
\text { MBA }\end{array}$ & $\begin{array}{l}\text { Montefiore } \\
\text { Medical Center, } \\
\text { Bronx, NY }\end{array}$ & $\begin{array}{l}\text { Designed and conceptualized } \\
\text { study, analyzed the data, } \\
\text { drafted the manuscript } \\
\text { for intellectual } \\
\text { content }\end{array}$ \\
\hline $\begin{array}{l}\text { David J. } \\
\text { Altschul, MD }\end{array}$ & $\begin{array}{l}\text { Montefiore } \\
\text { Medical Center, } \\
\text { Bronx, NY }\end{array}$ & $\begin{array}{l}\text { Designed and conceptualized } \\
\text { study, analyzed the data, drafted } \\
\text { the manuscript for intellectual } \\
\text { content }\end{array}$ \\
\hline $\begin{array}{l}\text { Rafael de La } \\
\text { Garza Ramos, } \\
\text { MD }\end{array}$ & $\begin{array}{l}\text { Montefiore } \\
\text { Medical Center, } \\
\text { Bronx, NY }\end{array}$ & $\begin{array}{l}\text { Interpreted the data, revised the } \\
\text { manuscript for intellectual } \\
\text { content }\end{array}$ \\
\hline $\begin{array}{l}\text { Phillip } \\
\text { Cezayirli, MD }\end{array}$ & $\begin{array}{l}\text { Montefiore } \\
\text { Medical Center, } \\
\text { Bronx, NY }\end{array}$ & $\begin{array}{l}\text { Interpreted the data, revised the } \\
\text { manuscript for intellectual } \\
\text { content }\end{array}$ \\
\hline $\begin{array}{l}\text { Santiago R. } \\
\text { Unda, MD }\end{array}$ & $\begin{array}{l}\text { Montefiore } \\
\text { Medical Center, } \\
\text { Bronx, NY }\end{array}$ & $\begin{array}{l}\text { Interpreted the data, revised the } \\
\text { manuscript for intellectual } \\
\text { content }\end{array}$ \\
\hline $\begin{array}{l}\text { Joshua Benton, } \\
\text { BA }\end{array}$ & $\begin{array}{l}\text { Montefiore } \\
\text { Medical Center, } \\
\text { Bronx, NY }\end{array}$ & Major role in acquisition of data \\
\hline $\begin{array}{l}\text { Joseph Dardick, } \\
\text { BA }\end{array}$ & $\begin{array}{l}\text { Montefiore } \\
\text { Medical Center, } \\
\text { Bronx, NY }\end{array}$ & Major role in acquisition of data \\
\hline $\begin{array}{l}\text { Aureliana } \\
\text { Toma, MD }\end{array}$ & $\begin{array}{l}\text { Montefiore } \\
\text { Medical Center, } \\
\text { Bronx, NY }\end{array}$ & Major role in acquisition of data \\
\hline $\begin{array}{l}\text { Nikunj Patel, } \\
\text { BA }\end{array}$ & $\begin{array}{l}\text { Montefiore } \\
\text { Medical Center, } \\
\text { Bronx, NY }\end{array}$ & Major role in acquisition of data \\
\hline $\begin{array}{l}\text { Avinash } \\
\text { Malaviya, BA, } \\
\text { MS }\end{array}$ & $\begin{array}{l}\text { Montefiore } \\
\text { Medical Center, } \\
\text { Bronx, NY }\end{array}$ & Major role in acquisition of data \\
\hline $\begin{array}{l}\text { David } \\
\text { Flomenbaum, } \\
\text { BS }\end{array}$ & $\begin{array}{l}\text { Montefiore } \\
\text { Medical Center, } \\
\text { Bronx, NY }\end{array}$ & Major role in acquisition of data \\
\hline $\begin{array}{l}\text { Jenelys } \\
\text { Fernandez- } \\
\text { Torres, BA }\end{array}$ & $\begin{array}{l}\text { Montefiore } \\
\text { Medical Center, } \\
\text { Bronx, NY }\end{array}$ & Major role in acquisition of data \\
\hline Jenny Lu, BA & $\begin{array}{l}\text { Montefiore } \\
\text { Medical Center, } \\
\text { Bronx, NY }\end{array}$ & Major role in acquisition of data \\
\hline $\begin{array}{l}\text { Ryan Holland, } \\
\text { MD }\end{array}$ & $\begin{array}{l}\text { Montefiore } \\
\text { Medical Center, } \\
\text { Bronx, NY }\end{array}$ & Major role in acquisition of data \\
\hline
\end{tabular}

Appendix (continued)

\begin{tabular}{|c|c|c|}
\hline Name & Location & Contribution \\
\hline $\begin{array}{l}\text { Elisabetta } \\
\text { Burchi, MD }\end{array}$ & $\begin{array}{l}\text { Montefiore } \\
\text { Medical Center, } \\
\text { Bronx, NY }\end{array}$ & $\begin{array}{l}\text { Interpreted the data, revised the } \\
\text { manuscript for intellectual } \\
\text { content }\end{array}$ \\
\hline $\begin{array}{l}\text { Richard } \\
\text { Zampolin, MD }\end{array}$ & $\begin{array}{l}\text { Montefiore } \\
\text { Medical Center, } \\
\text { Bronx, NY }\end{array}$ & Major role in acquisition of data \\
\hline Kevin Hsu, MD & $\begin{array}{l}\text { Montefiore } \\
\text { Medical Center, } \\
\text { Bronx, NY }\end{array}$ & Major role in acquisition of data \\
\hline $\begin{array}{l}\text { Andrew } \\
\text { McClelland, MD }\end{array}$ & $\begin{array}{l}\text { Montefiore } \\
\text { Medical Center, } \\
\text { Bronx, NY }\end{array}$ & Major role in acquisition of data \\
\hline $\begin{array}{l}\text { Judah Burns, } \\
\text { MD }\end{array}$ & $\begin{array}{l}\text { Montefiore } \\
\text { Medical Center, } \\
\text { Bronx, NY }\end{array}$ & Major role in acquisition of data \\
\hline $\begin{array}{l}\text { Amichai } \\
\text { Erdfarb, MD }\end{array}$ & $\begin{array}{l}\text { Montefiore } \\
\text { Medical Center, } \\
\text { Bronx, NY }\end{array}$ & Major role in acquisition of data \\
\hline $\begin{array}{l}\text { Rishi Malhotra, } \\
\text { MD }\end{array}$ & $\begin{array}{l}\text { Montefiore } \\
\text { Medical Center, } \\
\text { Bronx, NY }\end{array}$ & $\begin{array}{l}\text { Interpreted the data, revised the } \\
\text { manuscript for intellectual } \\
\text { content }\end{array}$ \\
\hline $\begin{array}{l}\text { Michelle Gong, } \\
\text { MD }\end{array}$ & $\begin{array}{l}\text { Montefiore } \\
\text { Medical Center, } \\
\text { Bronx, NY }\end{array}$ & $\begin{array}{l}\text { Interpreted the data, revised the } \\
\text { manuscript for intellectual } \\
\text { content }\end{array}$ \\
\hline $\begin{array}{l}\text { Peter Semczuk, } \\
\text { DDS, MPH }\end{array}$ & $\begin{array}{l}\text { Montefiore } \\
\text { Medical Center, } \\
\text { Bronx, NY }\end{array}$ & $\begin{array}{l}\text { Interpreted the data, revised the } \\
\text { manuscript for intellectual } \\
\text { content }\end{array}$ \\
\hline $\begin{array}{l}\text { Jonathan } \\
\text { Gursky, MD }\end{array}$ & $\begin{array}{l}\text { Montefiore } \\
\text { Medical Center, } \\
\text { Bronx, NY }\end{array}$ & Major role in acquisition of data \\
\hline
\end{tabular}

\begin{tabular}{|c|c|c|}
\hline $\begin{array}{l}\text { Victor } \\
\text { Ferastraoaru, } \\
\text { MD }\end{array}$ & $\begin{array}{l}\text { Montefiore } \\
\text { Medical Center, } \\
\text { Bronx, NY }\end{array}$ & Major role in acquisition of data \\
\hline $\begin{array}{l}\text { Jillian } \\
\text { Rosengard, MD }\end{array}$ & $\begin{array}{l}\text { Montefiore } \\
\text { Medical Center, } \\
\text { Bronx, NY }\end{array}$ & Major role in acquisition of data \\
\hline $\begin{array}{l}\text { Daniel } \\
\text { Antoniello, MD }\end{array}$ & $\begin{array}{l}\text { Montefiore } \\
\text { Medical Center, } \\
\text { Bronx, NY }\end{array}$ & Major role in acquisition of data \\
\hline $\begin{array}{l}\text { Daniel } \\
\text { Labovitz, MD }\end{array}$ & $\begin{array}{l}\text { Montefiore } \\
\text { Medical Center, } \\
\text { Bronx, NY }\end{array}$ & $\begin{array}{l}\text { Interpreted the data, revised the } \\
\text { manuscript for intellectual } \\
\text { content }\end{array}$ \\
\hline $\begin{array}{l}\text { Charles } \\
\text { Esenwa, MD }\end{array}$ & $\begin{array}{l}\text { Montefiore } \\
\text { Medical Center, } \\
\text { Bronx, NY }\end{array}$ & $\begin{array}{l}\text { Designed and conceptualized } \\
\text { study, analyzed the data, drafted } \\
\text { the manuscript for intellectual } \\
\text { content }\end{array}$ \\
\hline $\begin{array}{l}\text { Mark Milstein, } \\
\text { MD }\end{array}$ & $\begin{array}{l}\text { Montefiore } \\
\text { Medical Center, } \\
\text { Bronx, NY }\end{array}$ & $\begin{array}{l}\text { Designed and conceptualized } \\
\text { study, analyzed the data, } \\
\text { drafted the manuscript } \\
\text { for intellectual } \\
\text { content }\end{array}$ \\
\hline Alexis Boro, MD & $\begin{array}{l}\text { Montefiore } \\
\text { Medical Center, } \\
\text { Bronx, NY }\end{array}$ & $\begin{array}{l}\text { Designed and conceptualized } \\
\text { study, analyzed the data, drafted } \\
\text { the manuscript for intellectual } \\
\text { content }\end{array}$ \\
\hline $\begin{array}{l}\text { Mark F. Mehler, } \\
\text { MD }\end{array}$ & $\begin{array}{l}\text { Montefiore } \\
\text { Medical Center, } \\
\text { Bronx, NY }\end{array}$ & $\begin{array}{l}\text { Designed and conceptualized } \\
\text { study, analyzed the data, drafted } \\
\text { the manuscript for intellectual } \\
\text { content }\end{array}$ \\
\hline
\end{tabular}




\section{References}

1. Cheng Y, Luo R, Wang K, et al. Kidney disease is associated with in-hospital death of patients with COVID-19. Kidney Int 2020;97:829-838.

2. Yang X, Yu Y, Xu J, et al. Clinical course and outcomes of critically ill patients with SARS-CoV-2 pneumonia in Wuhan, China: a single-centered, retrospective, observational study. Lancet Respir Med 2020;8:475-481.

3. Ahmad I, Rathore FA. Neurological manifestations and complications of COVID-19: a literature review. J Clin Neurosci 2020;77:8-12.

4. Paniz-Mondolfi A, Bryce C, Grimes Z, et al. Central nervous system involvement by severe acute respiratory syndrome coronavirus-2 (SARS-CoV-2). J Med Virol 2020; 92:699-702.

5. Needham EJ, Chou SH, Coles AJ, Menon DK. Neurological implications of COVID19 infections. Neurocrit Care 2020;32:667-671.

6. Mao L, Jin H, Wang M, et al. Neurologic manifestations of hospitalized patients with coronavirus disease 2019 in Wuhan, China. JAMA Neurol 2020;77:683-690.

7. Asadi-Pooya AA, Simani L. Central nervous system manifestations of COVID-19: a systematic review. J Neurol Sci 2020;413:116832.

8. Jiménez-Ruiz A, García-Grimshaw M, Ruiz-Sandoval JL. Neurological manifestations of COVID-19. Gac Med Mex 2020;156.

9. Oxley TJ, Mocco J, Majidi S, et al. Large-vessel stroke as a presenting feature of COVID-19 in the young. N Engl J Med 2020;382:e60.

10. Al-Olama M, Rashid A, Garozzo D. COVID-19-associated meningoencephalitis complicated with intracranial hemorrhage: a case report. Acta Neurochir 2020;162:1495-1499.

11. Poyiadji N, Shahin G, Noujaim D, Stone M, Patel S, Griffith B. COVID-19-associated acute hemorrhagic necrotizing encephalopathy: CT and MRI features. Radiology 2020;296:E119-E120.

12. Yaghi S, Ishida K, Torres J, et al. SARS2-CoV-2 and stroke in a New York healthcare system. Stroke 2020;51:e316-e317.

13. Iadecola C, Anrather J, Kamel H. Effects of COVID-19 on the nervous system. Cell 2020;183:16-27.e1.

14. Todd A, Blackley S, Burton JK, et al. Reduced level of arousal and increased mortality in adult acute medical admissions: a systematic review and meta-analysis. BMC Geriatr 2017;17:283.

15. Sasson G, Bai AD, Showler A, et al. Staphylococcus aureus bacteremia in immunosuppressed patients: a multicenter, retrospective cohort study. Eur J Clin Microbiol Infect Dis 2017;36:1231-1241.
16. Altschul DJ, Unda SR, Benton J, et al. A novel severity score to predict inpatient mortality in COVID-19 patients. Sci Rep 2020;10:16726.

17. Altschul DJ, Unda SR, de La Garza Ramos R, et al. Hemorrhagic presentations of COVID-19: risk factors for mortality. Clin Neurol Neurosurg 2020;198: 106112.

18. Behman R, Nathens AB, Haas B, Look Hong N, Pechlivanoglou P, Karanicolas P. Surgery for adhesive small-bowel obstruction is associated with improved long-term survival mediated through recurrence prevention: a population-based, propensitymatched analysis. J Trauma Acute Care Surg 2019;87:636-644.

19. Matthiessen P, Hallböök O, Rutegård J, Sjödahl R. Population-based study of risk factors for postoperative death after anterior resection of the rectum. Br J Surg 2006; 93:498-503.

20. Varatharaj A, Thomas N, Ellul MA, et al. Neurological and neuropsychiatric complications of COVID-19 in 153 patients: a UK-wide surveillance study. Lancet Psychiatry $2020 ; 7: 875-882$.

21. Paterson RW, Brown RL, Benjamin L, et al. The emerging spectrum of COVID-19 neurology: clinical, radiological and laboratory findings. Brain 2020;143: 3104-3120.

22. Silva TJ, Jerussalmy CS, Farfel JM, Curiati JA, Jacob-Filho W. Predictors of in-hospital mortality among older patients. Clinics (Sao Paulo) 2009;64:613-618.

23. Nannoni S, de Groot R, Bell S, Markus HS. Stroke in COVID-19: a systematic review and meta-analysis. Int J Stroke Epub 2020Nov 11.

24. Amin R, Kitazawa T, Hatakeyama Y, et al. Trends in hospital standardized mortality ratios for stroke in Japan between 2012 and 2016: a retrospective observational study. Int J Qual Health Care 2019;31:G119-G125.

25. Kabarriti R, Brodin NP, Maron MI, et al. Association of race and ethnicity with comorbidities and survival among patients with COVID-19 at an urban medical center in New York. JAMA Netw Open 2020;3:e2019795

26. Gu T, Mack JA, Salvatore M, et al. COVID-19 outcomes, risk factors and associations by race: a comprehensive analysis using electronic health records data in Michigan Medicine. medRxiv 2020;3:e2025197.

27. Bhargava A, Sharma M, Riederer K, Fukushima EA, Szpunar SM, Saravolatz L. Risk factors for in-hospital mortality from COVID-19 infection among Black patients: an urban center experience. Clin Infect Dis Epub 2020 Sep 28.

28. Khazanchi R, Evans CT, Marcelin JR. Racism, not race, drives inequity across the COVID-19 continuum. JAMA Netw Open 2020;3:e2019933. 


\title{
Neurology
}

\author{
Neurologic Syndromes Predict Higher In-Hospital Mortality in COVID-19 \\ Emad Nader Eskandar, David J. Altschul, Rafael de la Garza Ramos, et al. \\ Neurology 2021;96;e1527-e1538 Published Online before print December 18, 2020 \\ DOI 10.1212/WNL.0000000000011356
}

This information is current as of December 18, 2020

Neurology ${ }^{\circledR}$ is the official journal of the American Academy of Neurology. Published continuously since 1951, it is now a weekly with 48 issues per year. Copyright Copyright (C) 2020 The Author(s). Published by Wolters Kluwer Health, Inc. on behalf of the American Academy of Neurology.. All rights reserved. Print ISSN: 0028-3878. Online ISSN: 1526-632X.

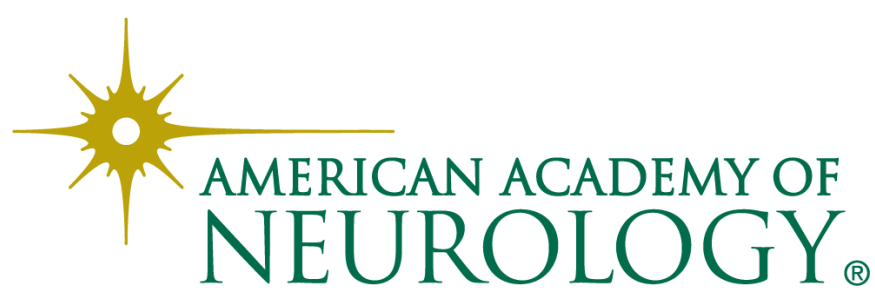




\section{Updated Information \& Services}

References

Citations

Subspecialty Collections

\section{Errata}

\section{Permissions \& Licensing}

\section{Reprints}

including high resolution figures, can be found at: http://n.neurology.org/content/96/11/e1527.full

This article cites 25 articles, 0 of which you can access for free at: http://n.neurology.org/content/96/11/e1527.full\#ref-list-1

This article has been cited by 2 HighWire-hosted articles: http://n.neurology.org/content/96/11/e1527.full\#\#otherarticles

This article, along with others on similar topics, appears in the following collection(s):

\section{All Cerebrovascular disease/Stroke}

http://n.neurology.org/cgi/collection/all_cerebrovascular_disease_strok e

All Epilepsy/Seizures

http://n.neurology.org/cgi/collection/all_epilepsy_seizures

\section{COVID-19}

http://n.neurology.org/cgi/collection/covid_19

Delirium

http://n.neurology.org/cgi/collection/delirium

Outcome research

http://n.neurology.org/cgi/collection/outcome_research

An erratum has been published regarding this article. Please see next page or:

/content/96/11/551.2.full.pdf

Information about reproducing this article in parts (figures,tables) or in its entirety can be found online at:

http://www.neurology.org/about/about_the_journal\#permissions

Information about ordering reprints can be found online:

http://n.neurology.org/subscribers/advertise

Neurology ${ }^{\circledR}$ is the official journal of the American Academy of Neurology. Published continuously since 1951, it is now a weekly with 48 issues per year. Copyright Copyright ( 2020 The Author(s). Published by Wolters Kluwer Health, Inc. on behalf of the American Academy of Neurology.. All rights reserved. Print ISSN: 0028-3878. Online ISSN: 1526-632X.

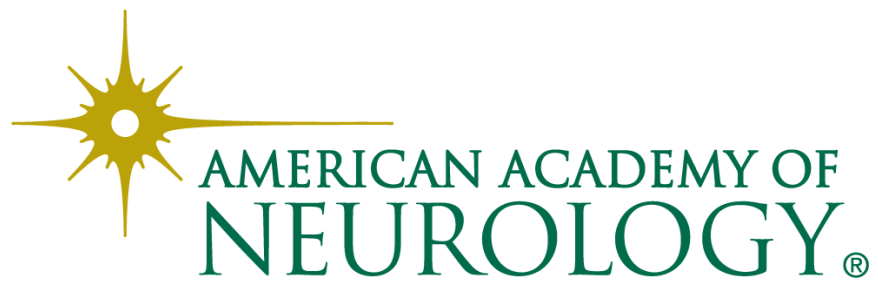




\title{
Disputes \& Debates: Editors' Choice
}

\author{
Steven Galetta, MD, FAAN, Section Editor
}

\section{Editor's Note: A Prospective Study of Neurologic Disorders in Hospitalized Patients With COVID-19 in New York City}

Dr. Frontera et al. examined the prevalence and associated mortality of well-defined neurologic diagnoses in a prospective, multicenter, observational study of 4,491 consecutive hospitalized adults in the New York City (NYC) metropolitan area with laboratory-confirmed Severe Acute Respiratory Syndrome Coronavirus 2 (SARS-CoV-2) infection. Neurologic disorders were diagnosed in $13.5 \%$ of these patients with coronavirus disease-2019 (COVID-19) and were associated with higher inhospital mortality and lower likelihood of discharge home. In response, Dr. Kumar et al. contrast the most common neurologic clinical diagnoses in this study (toxic/metabolic encephalopathy, stroke, seizure, and hypoxic/ischemic brain injury) with the most common imaging diagnoses in another study by Dr. Kremer et al. of patients with COVID-19 who underwent brain MRI (ischemic strokes, leptomeningeal enhancement, and encephalitis). They note that the study by Dr. Frontera et al. reported raised protein in the CSF in several of the patients, suggestive of intrathecal inflammation as may be seen with meningitis/encephalitis, although white cell counts were low. They wonder whether the low frequency of brain MRI in the study may have led to underdetection of meningitis/ encephalitis. In another response, Dr. Liotta et al. note that they reported similar rates of stroke, seizure, Guillain-Barre Syndrome, encephalitis, and meningitis in their recent Chicago-based study but had higher rates of encephalopathy. They note that in their own study, they adjudicated all charts, not just those for patients receiving neurologic consultations, and used protocolized delirium assessments to identify encephalopathy. They contend that Dr. Frontera et al. may have missed several cases of encephalopathy with their methodology and suggest that excluding headache as a neurologic symptom may have limited the scope of SARS-CoV-2 neuropathogenesis. They also note the lower in-hospital mortality in their cohort, potentially related to the absence of an overwhelming case surge in Chicago compared with NYC, and emphasize the importance of public health measures against COVID-19 to help sustain health care infrastructure. Responding to these comments, the authors question whether patients in the imaging study by Dr. Kremer et al. actually met accepted diagnostic criteria for encephalitis, noting that CSF SARS-CoV-2 RT-PCR (reverse transcription PCR) was negative in 20 patients and positing that some of the imaging findings could have represented postinfectious encephalitis. They also caution that many of the reported MRI findings are nonspecific and can be seen with nonencephalitic conditions, in particular hypoxic/ischemic injury, given the high frequency of acute respiratory distress syndrome and supplemental oxygen requirement among these patients. They also note that the elevated CSF protein in their own study is a nonspecific finding and highlight the need to follow rigorous standards when ascribing meningitis/ encephalitis to SARS-CoV-2 infection. Regarding their lower rates of encephalopathy compared with the Chicago study, the authors argue that they coded toxic-metabolic encephalopathy only in patients off sedation or after a sedation washout, whereas the Chicago study may have included patients with sedation-related delirium, which may have different outcomes than other etiologies of encephalopathy. However, they acknowledge that they may have underestimated the overall prevalence of neurologic injury in the most critically ill patients who could not be assessed off sedation, or who were unable to express other neurologic symptoms. Notwithstanding the older age of their cohort, they agree that the critical surge and strain on health care resources in NYC likely affected mortality and echo the importance of public health measures to stem such surges. This exchange demonstrates important differences that can arise in incidence or frequency estimates of different neurologic manifestations in COVID-19 based on the methodology that is followed.

Aravind Ganesh, MD, DPhil, FRCPC

Neurology ${ }^{\circledR}$ 2021;96:548. doi:10.1212/WNL.0000000000011604 


\section{Reader Response: A Prospective Study of Neurologic Disorders in Hospitalized Patients With COVID-19 in New York City}

Anand Kumar (Varanasi, India), Neha Lall (Varanasi, India), and Varun Kumar Singh (Varanasi, India) Neurology ${ }^{\circledR} 2021 ; 96: 549$. doi:10.1212/WNL.0000000000011614

We read with interest the article by Frontera et al. ${ }^{1}$ studying neurologic disorders in hospitalized COVID-19 patients in New York City. The overall prevalence of neurologic disorders among hospitalized COVID-19 patients was $13.5 \%$. The most common neurologic symptoms were toxic/metabolic encephalopathy $(309 / 606,51 \%)$, stroke $(84 / 606,14 \%)$, seizure $(74 / 606,12 \%)$, and hypoxic/ischemic brain injury $(65 / 606,11 \%)$. In a recent study by Kremer et al., ${ }^{2}$ correlated neurologic and neuroimaging findings of COVID-19 patients concluded that among 64 patients with neurologic symptoms who underwent brain MRI, ischemic strokes $(27 \%)$ were the most common finding, followed by leptomeningeal enhancement (17\%) and encephalitis (13\%). Even in present study, the CSF findings (table 2) show raised protein [median 61, IQR (42-106) $\mathrm{mg} / \mathrm{dL}$, favoring intrathecal inflammation and possibility of meningitis and/or encephalitis. Although there were few cells in the CSF [2 (1-4)], in COVID-19 patients, atypical inflammatory response without CSF pleocytosis is not uncommon. ${ }^{3}$ Another possible explanation of not picking up any encephalitis or meningitis in the present study is the lesser number of brain MRI (15\%) being performed. We are unable to understand the difference between stroke and hypoxic/ischemic brain injury because they were categorized separately in the current study!

1. Frontera JA, Sabadia S, Lalchan R, et al. A prospective study of neurologic disorders in hospitalized patients with COVID-19 in New York City. Neurology 2021;96:e575-e586.

2. Kremer S, Lersy F, Anheim M, et al. Neurologic and neuroimaging findings in patients with COVID-19. Neurology 2020;95: e1868-e1882.

3. Edén A, Kanberg N, Gostner J, et al. CSF biomarkers in patients with COVID-19 and neurological symptoms. Neurology 2020;96: e294-e300.

Copyright @ 2021 American Academy of Neurology

\section{Author Response: A Prospective Study of Neurologic Disorders in Hospitalized Patients With COVID-19 in New York City}

Jennifer A. Frontera (New York), Laura Balcer (New York), and Steven Galetta (New York)

Neurology ${ }^{\circledR}$ 2021;96:549-550. doi:10.1212/WNL.0000000000011609

We appreciate the comments by Kumar et al. on our article. ${ }^{1}$ The referenced Kremer study ${ }^{2}$ was a retrospective case series and included patients with positive MRI findings only. It is unclear whether the patients with the diagnosis of "encephalitis" met diagnostic or causal criteria outlined by the International Encephalitis Consortium ${ }^{3}$ and others. ${ }^{4}$ Indeed, CSF SARS-CoV-2 RT-PCR was negative in 20 patients. In 2 of 3 with CSF pleocytosis $\left(>5 \mathrm{cell} / \mathrm{mm}^{3}\right)$, imaging was performed $>2$ weeks from symptom onset, possibly representing postinfectious autoimmune encephalitis and not infectious encephalitis. Many of the MRI findings described are nonspecific and can be seen in hypoxic/ischemic brain injury, metabolic encephalopathy, or postseizure. Notably, $100 \%$ of "encephalitis" patients required oxygen and $75 \%$ had ARDS, suggesting that a proportion of the MRI changes may represent hypoxic/ischemic injury (defined as a global insult due to hypoxemia, hypotension, or cardiac arrest). Although we detected elevated CSF protein in some patients, ${ }^{1}$ this is nonspecific and can be found in stroke, hemorrhage (or traumatic tap), hypoxic/ischemic injury, diabetes, uremia, tumor, neuropathy, and many other conditions. Because the implications of SARS-CoV-2 neurotropism are far reaching, we believe that it is critical to follow the most rigorous standards and criteria when ascribing encephalitis/meningitis/myelitis to SARS-CoV-2 infection. 
1. Frontera JA, Sabadia S, Lalchan R, et al. A prospective study of neurologic disorders in hospitalized patients with COVID-19 in New York City. Neurology 2021;96:e575-e586.

2. Kremer S, Lersy F, Anheim M, et al. Neurologic and neuroimaging findings in patients with COVID-19. Neurology 2020;95: e1868-e1882.

3. Venkatesan A, Tunkel AR, Bloch KC, et al. Case definitions, diagnostic algorithms, and priorities in encephalitis: consensus statement of the international encephalitis consortium. Clin Infect Dis 2013;57:1114-1128.

4. Ellul MA, Benjamin L, Singh B, et al. Neurological associations of COVID-19. Lancet Neurol 2020;19:767-783.

\section{Reader Response: A Prospective Study of Neurologic Disorders in Hospitalized Patients With COVID-19 in New York City}

Eric M. Liotta (Chicago), Ayush Batra (Chicago), and Igor J. Koralnik (Chicago)

Neurology ${ }^{\circledR} 2021 ; 96: 550$. doi:10.1212/WNL.0000000000011613

Frontera et al. ${ }^{1}$ should be commended on the breadth of their report on neurologic diagnoses in COVID-19. Although stroke, seizure, GBS, encephalitis, and meningitis rates are similar to our recent study, ${ }^{2}$ and others, ${ }^{3,4}$ their rates of encephalopathy were markedly lower (6.9\% vs $31.8 \%) .{ }^{1,2}$ This likely reflects their methodology of adjudicating diagnoses only from chart review of patients with neurologic consultation. Our study similarly included patients with confirmed SARs-CoV-2 RT PCR and ascribed diagnoses by neurologist adjudication. We recognized that delirium - an entity within the encephalopathy spectrum - is the purview of multiple specialties. ${ }^{5}$ As such, encephalopathy would not reliably result in neurologic consultation; we adjudicated all charts and leveraged protocolized delirium assessments. The methodology of Frontera et al. likely failed to identify many encephalopathic patients, limiting their estimation of neurologic morbidity. Nevertheless, encephalopathy remained the most frequent neurologic diagnosis. In addition, prematurely excluding headache as a "neurologic symptom" limits the scope and understanding of SARS-CoV-2 neuropathogenesis. As we determine optimal management and decipher the long-term consequences of COVID-19 and encephalopathy, study methodologies should consider that not all neurologic complications result in inhospital neurologic consultation. Consistently, neurologic manifestations of COVID-19 are common and encephalopathy impacts morbidity. Interestingly, despite similar ventilation rates ( $26.3 \%$ vs $22.0 \%$ ), our cohort's hospital mortality was considerably lower ( $8.4 \%$ vs $21.4 \%){ }^{1,2}$ Although New York experienced a critical strain on hospital infrastructure early in the pandemic, our Chicago area hospital system never experienced the same overwhelming case surge. Taken together, Frontera et al. and our study may reflect the magnitude of public health benefit that could be realized by avoiding case volumes that overwhelm health care infrastructure. This should further emphasize the benefit of universal masking, social distancing, and building redundancy into health care infrastructure.

1. Frontera JA, Sabadia S, Lalchan R, et al. A prospective study of neurologic disorders in hospitalized patients with COVID-19 in New York City. Neurology 2021;96:e575-e586.

2. Liotta EM, Batra A, Clark JR, et al. Frequent neurologic manifestations and encephalopathy-associated morbidity in Covid-19 patients. Ann Clin Transl Neurol 2020;7:2221-2230.

3. Mao L, Jin H, Wang M, et al. Neurologic manifestations of hospitalized patients with coronavirus disease 2019 in Wuhan, China. JAMA Neurol 2020;77:683-690.

4. Romero-Sanchez CM, Diaz-Maroto I, Fernandez-Diaz E, et al. Neurologic manifestations in hospitalized patients with COVID-19: the ALBACOVID registry. Neurology 2020;95:e1060-e1070.

5. Slooter AJC, Otte WM, Devlin JW, et al. Updated nomenclature of delirium and acute encephalopathy: statement of ten Societies. Intensive Care Med 2020;46:1020-1022.

Copyright (c) 2021 American Academy of Neurology 


\section{Author Response: A Prospective Study of Neurologic Disorders in Hospitalized Patients With COVID-19 in New York City}

Jennifer A. Frontera (New York), Ariane Lewis (New York), Laura Balcer (New York), and

Steven Galetta (New York)

Neurology ${ }^{\circledR}$ 2021;96:551. doi:10.1212/WNL.0000000000011611

We appreciate these comments on our article. ${ }^{1}$ We coded toxic-metabolic encephalopathy only in patients off sedation or after an adequate sedation washout, in contrast to the Chicago study, ${ }^{2}$ which included patients who may have been receiving sedation or had a positive Confusion Assessment Method (CAM). Although sedation-related delirium has been associated with worse outcomes, the implications for long-term neurologic recovery differ based on the underlying etiologies of encephalopathy, which can best be ascertained when eliminating the confounding effect of sedative medications. Because a proportion of patients were too hypoxic for assessment off sedation, we recognize that we may be underestimating the overall prevalence of neurologic injury in the most critically ill patients. Similarly, hospitalized patients are often unable to express neurologic symptoms because of the severity of illness; hence, findings such as headache, anosmia, or dysgeusia are typically underrepresented and their prevalence is better studied in the outpatient setting. Although our cohort was somewhat older than the Chicago group-median age 65 vs 58 years-we agree that the critical surge and strain on resources in NYC likely impacted mortality rates, which were similarly high in other area hospitals during this time frame., ${ }^{3,4}$ Preventative efforts to stem such surges in hospitalizations_-including masking and social distancing—are essential.

1. Frontera JA, Sabadia S, Lalchan R, et al. A prospective study of neurologic disorders in hospitalized patients with COVID-19 in New York City. Neurology 2021;96:e575-e586.

2. Liotta EM, Batra A, Clark JR, et al. Frequent neurologic manifestations and encephalopathy-associated morbidity in Covid-19 patients. Ann Clin Transl Neurol 2020;7:2221-2230.

3. Cummings MJ, Baldwin MR, Abrams D, et al. Epidemiology, clinical course, and outcomes of critically ill adults with COVID-19 in New York City: a prospective cohort study. Lancet 2020;395:1763-1770.

4. Richardson S, Hirsch JS, Narasimhan M, et al. Presenting characteristics, comorbidities, and outcomes among 5700 patients hospitalized with COVID-19 in the New York city area. JAMA 2020;323:2052-2059.

Copyright (c) 2021 American Academy of Neurology

\section{CORRECTION}

\section{Neurologic Syndromes Predict Higher In-Hospital Mortality in COVID-19}

Neurology ${ }^{\circledR}$ 2021;96:551. doi:10.1212/WNL.0000000000011607

In the post-acceptance published version of the article "Neurologic Syndromes Predict Higher In-Hospital Mortality in COVID-19," by Nader Eskandar et al., ${ }^{1}$ an author was accidently omitted. The author byline and appendix should have included Jonathan Gursky, MD, from the Department of Neurology at Montefiore Medical Center, for his major role in acquisition of data. The omission is corrected in the final published version of the article. The authors regret the omission.

\section{Reference}

1. Nader Eskandar E, Altschul DJ, de La Garza Ramos R, et al. Neurologic syndromes predict higher in-hospital mortality in COVID-19. Neurology Epub 2020 Dec 18. 